medgen $2016 \cdot 28: 234-244$

DOI 10.1007/s11825-016-0089-z

Online publiziert: 15. August 2016

(c) Der/die Autor(en) 2016. Dieser Artikel ist eine Open-Access-Publikation.

CrossMark

Peter Ulz • Jochen B. Geigl • Michael R. Speicher - Ellen Heitzer

Institut für Humangenetik, Medizinische Universität Graz, Graz, Österreich

\title{
Neueste technologische Entwicklungen für die Analyse von zirkulierender Tumor-DNA
}

vollständig geklärt. Unklar ist, inwieweit ctDNA tatsächlich ein repräsentatives Porträt der Krebserkrankung darstellt, oder ob ctDNA nur die am meisten proliferierenden Klone reflektiert. Außerdem unterscheidet sich die Prävalenz der ctDNA in verschiedenen Tumorentitäten und selbst innerhalb einer Entität findet man eine erhebliche Variabilität [6]. Die Tatsache, dass zellfreie DNA (cfDNA) auch bei gesunden Individuen $\mathrm{zu}$ finden ist, spricht dafür, dass es sich bei der Freisetzung eigentlich um einen physiologischen Prozess handelt. Studien weisen aber darauf hin, dass man in bestimmten pathologischen Konditionen wie dem systemischen Lupus erythematodes (SLE), der rheumatoiden Arthritis und speziell bei Tumorpatienten erhöhte Mengen an zellfreier DNA findet [3, 6-13]. cfDNA wird unter physiologischen Bedingungen durch im Blut vorhandene DNAsen rasch abgebaut. Die Tatsache, dass bei Tumorpatienten häufig eine erniedrigte DNAse-Aktivität festgestellt wurde, ist eine Erklärung für die erhöhten Konzentrationen an cfDNA [12]. Aufgrund der charakteristischen Größenverteilung der zirkulierenden DNA-Fragmente von rund 166 bp, die exakt der Länge der DNA, die um ein Nukleosom gewickelt ist inklusive der linker region entspricht, ist es naheliegend anzunehmen, dass die Mehrheit der Fragmente von apoptotischen Zellen stammt [14, 15]. Sobald ein Tumor zu wachsen beginnt, erhöht sich nicht nur die Tumormasse, es kommt auch gleichzeitig zu einem Anstieg des Zellsterbens. Dadurch kann es zu einer verstärkten Freisetzung der Tumor-DNA kommen, woraus ein Ungleichgewicht zwischen der Ausbreitung und dem Abbau von cfDNA resultiert [16].

Studien zeigten, dass die cfDNA von unterschiedlichen Zellen freigesetzt werden kann [17, 18], wobei in der Regel der Hauptteil der cfDNA auf Zellen aus dem hämatopoetischen System zurückgeführt werden kann. Bei Personen mit Krebserkrankungen kann jedoch die ctDNA in unterschiedlichen Prozentsätzen in der cfDNA präsent sein. Zusätzlich zum zellulären Turnover hängt die Menge der im Blut vorhandenen ctDNA von einer Reihe weiterer Faktoren ab, wie beispielsweise dem Tumorstadium, der Anzahl und Lokalisation der Tumorherde, der Vaskularität oder dem Therapieansprechen und wahrscheinlich noch anderen, zurzeit unbekannten Faktoren. All diese Faktoren können zu einer immensen Variabilität der ctDNA beitragen. Sogar im metastasierten Krebsstadium kann der Anteil der Tumor-DNA im Plasma weniger als $1 \%$ bis zu mehr als $90 \%$ ausmachen [3, 6]. Die Diskriminierung der Tumor-DNA von DNA-Fragmenten, die von gesunden Zellen stammen, ist somit zusammen mit der starken Fragmentierung eine große Herausforderung im Bereich der ctDNA-Analytik.

In den letzten Jahren hat der technologische Fortschritt viele neue Methoden gebracht, die die nötige analytische Sensitivität und Spezifität aufweisen, um auch eine Detektion von stark unterrepräsentierten Allelen in der Zirkulation zu ermöglichen. Die meisten dieser sog. „High Resolution" Methoden sind zielgerichtet, d. h. sie beschränken sich auf die Analyse von einzelnen oder wenigen bekannten Mutationen oder Hotspots (• Abb. 2). In späteren Stadien verändern sich die 


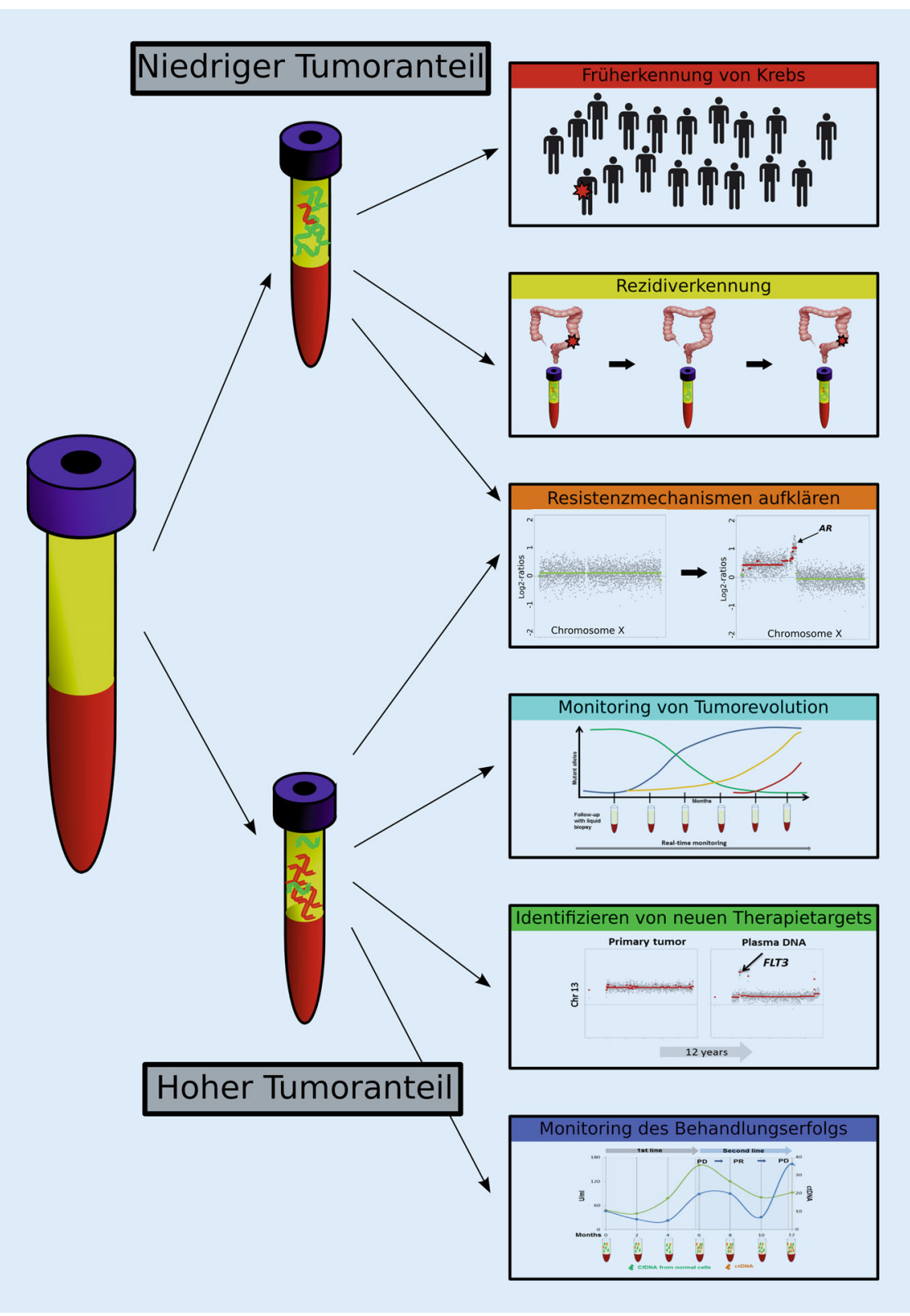

Abb. $1 \Delta$ Die Analyse zirkulierender Tumor-DNA (ctDNA) eröffnet vielerlei Möglichkeiten für den Einsatz in der Klinik. Für die Früherkennung von Tumoren bzw. Rezidiven sind hochauflösende Methoden notwendig, da die zu erwartende Menge an ctDNA gering ist. Das Monitoring der Tumorevolution, das Identifizieren von neuen Therapietargets sowie die Überwachung des Therapieerfolgs erfordern umfassendere Analysen und somit einen höheren Tumoranteil, da diese Methoden meist eine geringere analytische Sensitivität aufweisen. Bekannte Resistenzmechanismen können auch bei einem niedrigen Tumoranteil identifiziert werden, unbekannte hingegen erfordern genomweite Analysen, die bislang nur mit einem hohen Tumoranteil in der Probe möglich sind

Tumorgenome zunehmend und kontinuierlich im Rahmen ihrer Progression und des selektiven Drucks von Therapien. Hier sind nicht zielgerichtete Ansätze, die das Genom umfassend analysieren, notwendig, um die Dynamik des Tumorgeschehens und neu auftretende Veränderungen im Tumorgenom kontinuierlich erfassen zu können (• Abb. 2). die Analyse von ctDNA sowie deren potenzielle Anwendungen diskutiert (• Tab. 1).

\section{Hochauflösende Methoden zu Detektion von unterrepräsen- tierten Allelen}

In den letzten Jahren konnten in der Analyse von ctDNA, betreffend die analytische Sensitivität und Spezifität, erhebliche Fortschritte gemacht werden. Die erhöhte Sensitivität kann man einerseits auf den Einsatz von modifizierten Polymerasen mit einer reduzierten Fehlerrate sowie auf die Verwendung von digitalen Methoden und ausgefeilten bioinformatischen Algorithmen zurückführen. Seit den ersten zielgerichteten $\mathrm{Mu}-$ tationsanalysen in Plasma bzw. Serum in den 1990ern hat der technologische Fortschritt eine ganze Reihe von hoch sensitiven Methoden wie beispielsweise ARMS [22], digitale PCR [23, 24] oder BEAMing [25] mit sich gebracht, mit denen es heute möglich ist, Mutationen auch weit unter $1 \%$ zu detektieren. Mittels ARMS-PCR und anderer allelspezifischer Ansätze ist es zum Bespiel möglich, Hotspot-Mutationen in KRAS, BRAF, und EGFR mit einem Detektionslimit (LOD, engl. limit of detection) von $0,001-2 \%$ nachzuweisen [22, 26-30].

Die castPCR (competitive allele-specific TaqMan PCR) basiert auf einem ähnlichen Ansatz, bei dem die Wildtyp-Allele blockiert und die Zielmoleküle präferenziell amplifiziert werden, und erlaubt somit eine Detektion von bis zu 0,1 \% mutierter DNA [31]. In einem direkten Vergleich zwischen dem Therascreen ARMS assay (Qiagen) - ein von der FDA zugelassener diagnostischer Test für die Detektion von EGFR- und KRAS-Mutationen aus FFPE-Material - und der castPCR (Life Technologies) für KRAS, erreichte die castPCR eine vergleichbar gute Sensitivität und Spezifität [32]. Eine ganze Reihe von ähnlichen, etwas exotischeren Anwendungen wurde bereits publiziert, allerdings hat bisher keine davon eine breite Anwendung gefunden [33-48].

Trotz der riesigen Auswahl an hochauflösenden Methoden scheinen sich digitale Verfahren durchzusetzen $[49,50]$. Die digitale PCR (APCR) beruht auf ei- 
ner Verdünnung und Partitionierung der zu analysierenden Proben in Einzelmoleküle, die klonal amplifiziert und separat detektiert werden. Zwei unterschiedliche Ansätze für die dPCR werden zurzeit angeboten: Fluidigm sowie Thermo Fisher (OpenArray, QuantStudio) partitionieren die individuellen Reaktionen in $\mathrm{Mi}$ krofluidik Reaktionskammern, während RainDance Technologies und Biorad die Moleküle in einer Wasser-in-Öl-Emulsion vereinzeln. Diese Technologien werden häufig auch als digital droplet PCR (ddPCR) bezeichnet. Die klonale Amplifikation kann aber auch an magnetischen Beads erfolgen, wie bei der BEAMingMethode (beads, emulsions, amplification and magnetics) [23, 25, 51]. Mit einer dPCR kann man analytische Sensitivitäten von $0,01-0,0005 \%$ erzielen [24, $50,52,53]$. In diesem Kontext muss erwähnt werden, dass diese Sensitivitäten nur durch ausreichende Ausgangsmengen an DNA gewährleistet werden können, was in vielen Fällen für cfDNA nicht möglich ist.

Eine ebenfalls hoch sensitive und vor allem auch spezifische Methode ist die Identifizierung von chromosomalen Umbauten, wie beispielsweise Translokationen, aus dem Primärtumor, mit einer nachfolgenden Detektion aus Plasma mithilfe von dPCR [54, 55]. Fusionsbruchpunkte können somit in der Blutzirkulation bis zu einem LOD von $0,001 \%$ nachgewiesen werden, wodurch sich dieser Ansatz speziell für die Identifizierung von MRD und die Früherkennung von Rezidiven eignet. Eine breite Anwendung ist im Moment aber noch ausgeschlossen, da der Identifizierung von Bruchpunkten eine Genomsequenzierung (WGS, engl. whole genome sequencing) vorausgeht, welche im Moment noch zu kosten- und zeitintensiv ist.

\section{Next Generation Sequencing basierte Methoden und Unterdrückung der Fehlerraten}

Der Vorteil von NGS (engl. next generation sequencing)-basierten Methoden liegt darin, dass umfassendere Analysen von mehreren Genen oder genomischen Regionen möglich sind. Konven-

medgen 2016 $28: 234-244$ DOI 10.1007/s11825-016-0089-z

๑ Der/die Autor(en) 2016. Dieser Artikel ist eine Open-Access-Publikation.

P. Ulz · J. B. Geigl · M. R. Speicher · E. Heitzer

\section{Neueste technologische Entwicklungen für die Analyse von zirkulierender Tumor-DNA}

\section{Zusammenfassung}

Die Analyse von zirkulierender Tumor-DNA, zusammen mit der Analyse von zirkulierenden Tumorzellen auch oft Liquid Biopsy genannt, ist ein sich rasch entwickelndes Feld in der medizinischen Forschung. Obwohl es von der Entdeckung der zellfreien DNA bis hin zur Erkenntnis, dass sie sich als Biomarker eignet, Jahrzehnte gedauert hat, wurde der klinische Nutzen der ctDNA hinsichtlich der Überwachung des Therapieansprechens, der Identifizierung von Resistenzmechanismen und neu aufkommenden Therapiezielen sowie der Detektion von minimaler Resterkrankung mittlerweile in unzähligen Studien bewiesen.
Aufgrund der hohen Variabilität, mit der ctDNA in der Zirkulation vorkommt, sowie der starken Fragmentierung, stellt die ctDNA aber einen schwierigen Analyten dar. In den letzten Jahren haben erhebliche technologische Fortschritte dazu beigetragen, dass eine Routineanwendung der ctDNA-Analysen tatsächlich realisierbar wird, sofern eine Reihe von regulatorischen Hürden überwunden wird.

Schlüsselwörter Liquid Biopsy · CtDNA-Analysen - Biomarker . Surrogatmarker - Tumordiagnostik . Zirkulierende Tumorzellen

\section{Latest technological developments in the analysis of circulating tumor DNA}

\section{Abstract}

The analysis of circulating tumor DNA (ctDNA) and circulating tumor cells, often known as liquid biopsy, is a rapidly developing field of medical research. Although it has taken decades since the discovery of cellfree DNA for it to be recognized as a suitable biomarker, the clinical benefit of ctDNA with regard to monitoring therapy response, the identification of resistance mechanisms, and novel emerging actionable targets, in addition to the detection of minimal residual disease, has recently been proven in numerous studies.
Owing to the great variability of ctDNA in the circulation, together with the high degree of fragmentation, ctDNA is a challenging analyte. However, in recent years technological advances have contributed to a variety of routine applications of ctDNA analysis becoming a reality, given that a number of additional regulatory hurdles can be overcome.

\section{Keywords}

Liquid biopsies - CtDNA analysis - Biomarker . Surrogate marker - Clinical oncology . Circulating tumor cells tionelle NGS-Methoden sind allerdings auf eine Sensitivität zwischen 1-5 \% limitiert, da während Amplifikation und Sequenzierung aufgrund mangelnder Genauigkeit der Polymerasen PCR-Fehler oder Sequenzierartefakte in die DNA eingeführt werden können. Durch die starke Fragmentierung und die limitierten Ausgangsmengen an cfDNA wird die Situation noch erschwert, da zumeist die Anzahl der PCR-Zyklen in der Library Präparation im Vergleich zu genomischen DNAProben erhöht werden muss, was wiederum zu einer Erhöhung der Fehlerrate führt.
In den letzten Jahren wurden unterschiedliche Ansätze entwickelt, um die Fehlerrate zu minimieren. Ein Algorithmus, entwickelt von Narayan et al., in dem eine Konsensussequenz nach einer Paired-End-Sequenzierung über den überlappenden Bereich gebildet wird, konnte die Fehlerrate von $0,31 \%$ pro Base auf $0,07 \%$ verringern [56]. Mit demselben Ansatz konnte eine japanische Gruppe LODs von 0,01, 0,01 und $0,05 \%$ für die Detektion der EGFR Hotspot Mutationen L858R, L861Q, und T790M aus Plasma erzielen [57, 58].

Eine weitere Möglichkeit, die Fehlerrate $\mathrm{zu}$ reduzieren, stellen molekulare 
Barcoding Strategien dar. Dabei wird bei der PCR jedes Ausgangsmolekül mit einem sog. Unique Identifier (UID), einer kurzen degenerierten Sequenz markiert, die es erlaubt, nach der Sequenzierung alle Tochterstränge zu einer Konsensussequenz zusammenzufassen. Mutationen müssen demnach in allen Sequenzen der Tochterstränge vorkommen, während Artefakte nur in einem Teil der Fragmente zu finden sind. Dieser Ansatz wurde 2011 erstmals von der Vogelstein-Gruppe unter dem Namen SafeSeqS publiziert und findet eine immer breitere Anwendung [59-61]. Kinde et al. konnten durch diese Strategie die Fehlerrate für einzelne Targets um das 15- bis 24-Fache reduzieren. Mit einer Kombination von molekularem Barcoding und ausgefeilten bioinformatischen Algorithmen (iDES, digital error suppression) konnten Newman et al. die Fehlerrate pro Base auf $9 \times 10^{-5}$ verringern [62] Im Gegensatz zur Safe-SeqS, bei der UIDs über eine PCR angefügt werden, werden die Barcodes beim sog. CAPPSeq im Rahmen der Library Präparation ligiert. Mittels einer anschließenden hybridisierungsbasierten Anreicherung von Zielregionen ist es möglich, mehrere Gene gleichzeitig zu analysieren. Die Firma Guardant Health, Inc. (http://www. guardanthealth.com/guardant360/) bietet mit dem Digital Sequencing ${ }^{\mathrm{TM}}$ eine ähnliche Methode an, mit der nach Sequenzierung von 54 klinisch relevanten Targets eine Auflösung von 0,1 \% mutierter Allelfrequenz bei einer analytischen Spezifität von mehr als 99,99\% erreicht wird [63].

\section{Panel Sequenzierung}

Obwohl die zielgerichtete Analyse von einzelnen oder wenigen Targets eine hohe Auflösung erreichen kann, ist in vielen Szenarien auch eine umfassendere Analyse von Bedeutung, insbesondere wenn neue Veränderungen, die während der Tumorevolution entstehen können, erfasst werden sollen. Wenn die zu analysierenden Targetregionen auf eine Größe von einigen hundert Kilobasen beschränkt sind, kann eine amplikonbasierte Anreicherung eine kostengünstige Variante darstellen. Für größere Regionen
Hier steht eine Anzeige.

를 Springer 


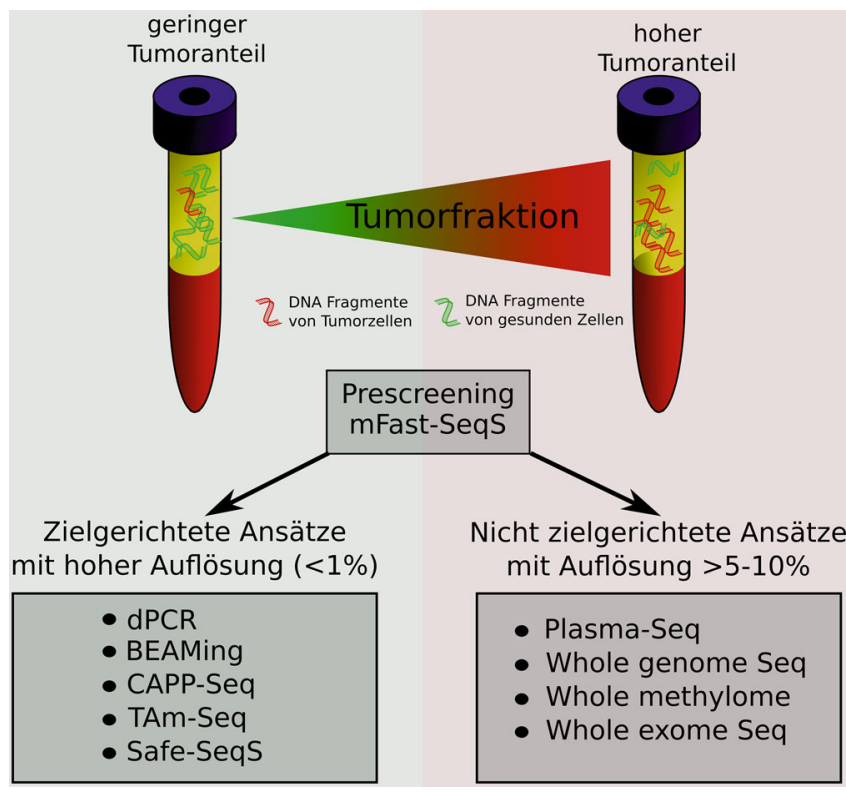

Abb. $2 \Delta$ Der Anteil an zirkulierender Tumor-DNA im Vergleich zu zellfreier DNA aus normalen Zellen ist entscheidend für die Auswahl der Analysemethoden. Für Proben mit geringen Mengen an Tumor-DNA $(<1 \%)$ sind gezielte, hochauflösende Methoden notwendig, um die durch gesunde DNA verdünnte Mutation noch nachzuweisen. Wenn ausreichende Mengen an Tumor-DNA in der Zirkulation vorhanden sind (>5-10\%), können auch genomweite Methoden mit geringerer analytischer Sensitivität angewandt werden

ist allerdings eine hybridisierungsbasierte Anreicherung anzuraten.

Im klinischen Umfeld wird zumeist die Analyse von bekannten prädiktiven bzw. therapierbaren Alterationen favorisiert. Das National Comprehensive Cancer Network (NCCN) empfiehlt beispielsweise für die Auswahl zwölf zielgerichteter Therapien für nicht kleinzellige Lungentumoren (NSCLC, engl. non-small cell lung cancer) die Analyse von sieben Genen (EGFR, $A L K$, ERBB2/HER2, BRAF, MET, ROS1 und $R E T$ ) [64]. Eine relativ aktuelle Studie konnte zeigen, dass nach Analyse von 25 sog. Driver-Genen, also Genen, die an Initiierung und Progression des Tumors beteiligt sind, in zehn unterschiedlichen Tumorentitäten zumindest jeweils eine relevante Mutation detektiert werden konnte (Sensitivität 65-77\%) [65]. Obwohl im Bereich der Pathologie eine ganze Reihe von kommerziell erhältlichen sog. Hotspot Panels in Verwendung sind (AmpliSeq Cancer Panel, Life Tech; TruSeq Cancer Amplicon, Illumina; TruSight 15, Illumina; the ThunderBolts $\mathrm{s}^{\mathrm{m}}$ Cancer Panel, Raindance), gibt es auf- grund technischer Limitationen kaum Daten für cfDNA [66]. In einer kürzlich veröffentlichen Studie wurde nach der Analyse des AmpliSeq Cancer Panel in Plasma-DNA und korrespondierenden Tumorproben eine Konkordanz von 76 \% gezeigt [67]. Eine weitere Studie erzielte zwar eine hohe Spezifität (>95\%), allerdings lag die Sensitivität bei Verwendung desselben Panels nur bei $55 \%$. Eine suboptimale Detektionsrate der Mutation aufgrund niedriger Ausgangsmengen in der Plasma DNA, das Vorliegen der Mutationen mit Allelfrequenzen, die unter der Nachweisgrenze liegen, sowie schlechte DNA-Qualität der Tumorproben und Tumorheterogenität wurden als mögliche Faktoren für diese Ergebnisse angeführt [68]. Aus diesem Grund haben verschiedene Gruppen eigene Panels, die besser für die Analyse von cfDNA geeignet sind, entwickelt. TAmSeq (tagged-amplicon deep sequencing), etabliert von der Rosenfeld-Gruppe in Cambridge, vereint beispielsweise eine Präamplifikation der Zielregionen im Multiplexansatz mit einer anschließenden selektiven Amplifikation der Region in Singleplex, um nicht spezifische PCRProdukte zu vermeiden [69, 70]. Dabei kann ein LOD von $2 \%$ bei einer Sensitivität und Spezifität von $>99 \%$ erreicht werden. Die zu analysierenden Zielregionen können einfach und rasch für unterschiedliche Tumorentitäten angepasst werden, wodurch eine große Flexibilität für das Design von personalisierten Assays gegeben ist [69].

Eine Limitation von PCR-basierten Ansätzen ist die Tatsache, dass man damit keine komplexen genomischen Alterationen detektieren kann. Ein kostengünstigerer Lösungsansatz als ein WGS ist hierfür die spezifische Anreicherung von Regionen, die in chromosomalen Umbauten involviert sind [21, 71]. In einer aktuellen Studie, in der eine hybridisierungsbasierte Anreicherung mit inkludierten molekularen Barcodes von kodierenden sowie von in Translokationen involvierten Regionen aus PlasmaDNA von NSCLC-Patienten durchgeführt wurde, konnte eine diagnostische Sensitivität und Spezifität von $100 \%$ bei einem Detektionslimit für mutierte Sequenzen von $0,4 \%$ erreicht werden [72]. Eine andere Gruppe machte sich öffentlich zugängliche Sequenzierdaten von Lungentumoren zunutze, um ein Panel zu designen, mit dem in mehr als $90 \%$ der Patienten zumindest eine Mutation detektiert werden kann [73]. Durch das Hinzufügen von molekularen Barcodes konnte deren ursprünglich publizierter CAPP-Seq Ansatz hinsichtlich der analytischen Sensitivität massiv verbessert werden, wobei eine hypothetische Sensitivität von $0,0025 \%(2,5$ in $10^{5}$ Molekülen) angegeben wurde [62]. Da eine solch hohe Sensitivität nur durch die Detektion von mehreren Mutationen erzielt werden kann, haben die Autoren das Panel optimiert, sodass bei zumindest $50 \%$ der Patienten mindestens acht Mutationen nachgewiesen werden können.

\section{Exome sequencing}

Die erste Exomsequenzierung (WES, whole exome sequencing) aus PlasmaDNA wurde 2013 berichtet; dabei wurden Patienten über mehrere Therapielinien verfolgt, wobei jeweils zum Zeitpunkt 


\begin{tabular}{|c|c|c|c|c|}
\hline Methode & Vorteile & Nachteile & LOD (\%) & Referenzen \\
\hline ARMS & $\begin{array}{l}\text { Hohe analytische Sensitivität } \\
\text { \& Spezifität, günstig }\end{array}$ & $\begin{array}{l}\text { Mutation muss bekannt sein, } \\
\text { nur einzelne, wenige Zielregionen } \\
\text { analysierbar }\end{array}$ & Bis zu 0,001 & {$[22,26-30]$} \\
\hline $\mathrm{dPCR}$ & $\begin{array}{l}\text { Hohe analytische Sensitivität } \\
\text { \& Spezifität }\end{array}$ & $\begin{array}{l}\text { Mutation muss bekannt sein, } \\
\text { nur einzelne, wenige Zielregionen } \\
\text { analysierbar, } \\
\text { spezielles Equipment erforderlich, teuer }\end{array}$ & Bis zu 0,0005 & {$[23,24,50-53]$} \\
\hline BEAMING & $\begin{array}{l}\text { Hohe analytische Sensitivität } \\
\text { \& Spezifität }\end{array}$ & $\begin{array}{l}\text { Mutation muss bekannt sein, } \\
\text { nur einzelne, wenige Zielregionen } \\
\text { analysierbar, } \\
\text { spezielles Equipment erforderlich, teuer }\end{array}$ & 0,001 & [25] \\
\hline castPCR & $\begin{array}{l}\text { Gute analytische Sensitivität } \\
\& \text { Spezifität }\end{array}$ & $\begin{array}{l}\text { Mutation muss bekannt sein, } \\
\text { nur einzelne, wenige Zielregionen } \\
\text { analysierbar }\end{array}$ & 0,10 & [31] \\
\hline $\begin{array}{l}\text { Detektion von } \\
\text { Fusionsbruchpunkten mit } \\
\text { dPCR }\end{array}$ & Sehr gute Spezifität & $\begin{array}{l}\text { Identifizierung der Bruchpunkte } \\
\text { aufwendig, } \\
\text { teuer }\end{array}$ & 0,001 & {$[54,55]$} \\
\hline $\begin{array}{l}\text { Bioinformatische Error } \\
\text { Suppression }\end{array}$ & $\begin{array}{l}\text { Verbesserung der Fehlerrate } \\
\text { nach Sequenzieren }\end{array}$ & Limitierte Auflösung & 0,01 & {$[56-58]$} \\
\hline Molekulares Barcoding & $\begin{array}{l}\text { Verbesserung der Fehlerrate } \\
\text { nach Sequenzieren }\end{array}$ & $\begin{array}{l}\text { Verringerte PCR Effizienz, } \\
\text { hohe Sequenzierkapazität notwendig }\end{array}$ & 0,01 & [62] \\
\hline Cancer Hotspot Panels & $\begin{array}{l}\text { Analyse von klinisch } \\
\text { relevanten Targets }\end{array}$ & Fehlende analytische Sensitivität & $1-5$ & - \\
\hline TAm-Seq & $\begin{array}{l}\text { Hohe Flexibilität für } \\
\text { personalisierte Assays }\end{array}$ & Fehlende analytische Sensitivität & 2 & {$[69,70]$} \\
\hline CAPP-Seq & $\begin{array}{l}\text { Hohe diagnostische } \\
\text { Sensitivity }\end{array}$ & Aufwendige Analytik & 0,40 & [72] \\
\hline CAPP-Seq \& iDES & $\begin{array}{l}\text { Hohe analytische und } \\
\text { diagnostische Sensitivität }\end{array}$ & $\begin{array}{l}\text { Hohe analytische und diagnostische } \\
\text { Sensitivität }\end{array}$ & 0,0025 & [62] \\
\hline Whole exome sequencing & Umfassende Analyse & $\begin{array}{l}\text { Fehlende analytische Sensitivität, } \\
\text { keine SCNAs }\end{array}$ & 5 & [74-76] \\
\hline Array-CGH & $\begin{array}{l}\text { Umfassende Analyse, } \\
\text { Bestimmung von SCNA }\end{array}$ & $\begin{array}{l}\text { Fehlende analytische Sensitivität, } \\
\text { keine Mutationsdaten }\end{array}$ & 10 & [14] \\
\hline SNP-Arrays & $\begin{array}{l}\text { Umfassende Analyse, } \\
\text { Bestimmung von SCNA }\end{array}$ & $\begin{array}{l}\text { Fehlende analytische Sensitivität, } \\
\text { keine Mutationsdaten }\end{array}$ & 10 & [85] \\
\hline Low coverage WGS & $\begin{array}{l}\text { Umfassende Analyse, } \\
\text { Bestimmung von SCNA }\end{array}$ & $\begin{array}{l}\text { Fehlende analytische Sensitivität, } \\
\text { keine Mutationsdaten }\end{array}$ & $5-10$ & {$[21,75,86,87]$} \\
\hline mFAST-Seqs & $\begin{array}{l}\text { Umfassende Analyse, } \\
\text { Bestimmung von SCNA }\end{array}$ & $\begin{array}{l}\text { Fehlende analytische Sensitivität, } \\
\text { keine Mutationsdaten }\end{array}$ & $5-10$ & [88] \\
\hline
\end{tabular}

der Progression ein WES durchgeführt wurde, während zu den dazwischenliegenden Zeitpunkten die identifizierten Mutationen mit hochauflösenden Methoden gescreent wurden [74]. Mittels dieser Strategie konnten bei allen $\mathrm{Pa}$ tienten resistenzassoziierte Mutationen nachgewiesen werden [74]. Obwohl WES auch in zwei weiteren Studien angewandt wurde, ist es aufgrund der Kosten, der geringen Sensitivität bzw. der umfassenden Interpretation der Varianten wohl nicht die geeignetste Methode für ein Therapiemonitoring [75, 76]. Hingegen erweist sich ein WES aus Tumormaterial für die Identifizierung von patientenspezifischen Mutationen als nützlich, welche dann für ein hochauflösendes, longitudinales Monitoring im Plasma herangezogen werden können [77, 78].

\section{Umfassende genomweite Ansätze}

Speziell in späten Stadien unterliegen Tumorgenome einer immensen Dynamik. Die genetische Landschaft von Tumoren und/oder Metastasen kann sich rasch, und in manchen Fällen gravierend, verändern, sodass nicht zielgerichtete, genomweite Ansätze notwendig sind, um diese Änderung zu erfassen [19, 21, 79, 80]. Außerdem erlauben solche Metho- 
den unter anderem eine genomweite $\mathrm{Be}$ stimmung von somatischen Kopienzahlveränderungen (SCNA, engl. somatic copy number alteration), die kennzeichnend für $90 \%$ der soliden bzw. $50 \%$ der hämatologischen Malignitäten sind [81]. Zudem sind Onkogene häufig von Änderungen der Kopienzahl betroffen und eine erhöhte Anzahl an SCNAs ist mit einer schlechteren Prognose assoziiert [82-84]. Kopienzahlanalysen, die traditionell mittels Array-CGH oder SNPArrays bestimmt wurden, werden seit Einführung des NGS mehr und mehr durch WGS abgelöst [14, 85]. Dabei wird das Genom virtuell in definierte Abschnitte unterteilt, in denen dann die generierten Sequenzinformationen gezählt und mit einem Erwartungswert bzw. einer Kontrollpopulation verglichen werden. Die Etablierung von SCNAs aus Plasma anhand von low-coverage WGS, wie beispielsweise die von uns etablierte plasma-Seq Technologie, hat sich als eine äußerst effektive Methode erwiesen, um rasch und kostengünstig die Plastizität und Evolution von Tumoren zu erfassen [21, 75, 86, 87]. Das erste WGS aus Plasma wurde 2012 publiziert [55]. In den folgenden Jahren wurden dann weitere Ansätze, hauptsächlich von uns und der Gruppe um Dennis Lo, etabliert [19, 21, 75, 79, 86, 87]. Mittels einer kombinierten Analyse von SCNAs und Hypomethylierung konnte die LoGruppe eine diagnostische Sensitivität und Spezifität von $74 \%$ bzw. $94 \%$ im Vergleich zu den korrespondierenden Tumorproben erzielen [79].

Wie auch bei der WES ist die fehlende analytische Sensitivität bei diesen Analysen die größte Limitation [3]. Mit unserer plasma-Seq Methode ist es möglich, SCNAs mit einer Sensitivität und Spezifität von $80 \% \mathrm{zu}$ detektieren, wenn $5-10 \%$ der cfDNA im Plasma vom Tumor stammt. Allerdings kann es sogar bei hochmetastasierten Patienten aufgrund der im ersten Abschnitt dargelegten Parameter vorkommen, dass dieser Cut-Off nicht erreicht wird [6, 21, 55,75 ] und bei rund 20-30\% der Patienten können mit diesen Methoden keine informativen Ergebnisse erzielt werden. Um jene Patienten vorab zu identifizieren, bei denen ausreichend Tumor-DNA für genomweite Analysen in der Zirkulation vorhanden ist, haben wir kürzlich eine pre-screening Methode entwickelt [88]. Das sog. mFAST-SeqS basiert auf einer selektiven Amplifikation von LINE1-Sequenzen, die im gesamten Genom verteilt vorliegen. [88]. Ähnlich wie bei der genomweiten Kopienzahlanalyse werden die generierten LINE1-Sequenzen gegen das Genom kartiert und pro Chromosomenarm gezählt. Das Ausmaß einer möglichen Abweichung wird durch den Vergleich mit Kontrollproben in einem z-Score zum Ausdruck gebracht. Die Summe aller chromosomenarmspezifischen $\mathrm{z}$-Scores ist ein generelles Maß für Aneuploidie und reflektiert den Anteil der tumorspezifischen DNA in der Probe [88].

\section{Potenzielle klinische Anwen- dungen von hochauflösenden Methoden}

Die gezielte Analyse von bekannten prädiktiven Targets sowie die frühzeitige Identifizierung von Resistenzmechanismen aus Plasma ist zur Zeit die Anwendung, die am ehesten ihre Verwendung in der Klinik findet. Allerdings bedarf es hier hochauflösender Methoden, damit etwaige Resistenzmutationen frühzeitig auch bei geringen Tumorfraktionen im Plasma detektiert werden können.

Speziell bei Kolon- und Lungenkarzinomen bekommt die molekulargenetische Charakterisierung der Tumore immer mehr Bedeutung, um beispielsweise das Ansprechen auf EGFR-gerichtete Therapien vorherzusagen [89]. Lungentumore, die aktivierende Mutationen im EGFR-Gen tragen, sprechen in der Regel gut auf eine Klasse von Tyrosinkinaseinhibitoren (TKI) an [90]. Allerdings kommt es bei fast allen Patienten nach einem initial guten Ansprechen innerhalb von zwölf Monaten zur Resistenz [91]. Die Resistenz ist in den meisten Fällen ( $60 \%)$ auf das Auftreten einer sekundären EGFR-Mutation (T790M) zurückzuführen. Da mittlerweile eine zweite Generation von TKIs auf dem Markt ist, die auch bei Vorhandensein der Resistenzmutation zu einer EGFR-Blockade führen, ist die frühzeitige Identifizierung der Resistenzmutation von großem klini- schen Interesse [92, 93], wobei die Analyse von ctDNA aus Plasma hier immenses Potenzial zeigt. In einer bereits 2011 veröffentlichten Studie konnte mit der BEAMing-Methode bei $43 \%$ der resistenten Patienten die T790M-Mutation im Plasma nachgewiesen werden [94]. Eine aktuelle Studie erzielte unter Verwendung von ddPCR einen positiven prädiktiven Wert von $100 \%$ für zwei aktivierende EGFR-Mutationen (19 del, L858R) sowie $74 \%$ für die Detektion von T790M [95].

Ein überaus wichtiger Punkt ist zudem, dass Resistenzmutationen im Plasma auch dann nachgewiesen werden können, wenn eine zeitgleiche Biopsie nicht möglich ist oder ein negatives Ergebnis liefert [96, 97]. Der Nachweis der T790M-Mutation aus Plasma kann somit in vielen Fällen ein Therapieversagen vorhersagen, lange bevor eine klinische Progression evident ist, woraufhin die Therapie frühzeitig adaptiert werden kann.

Da Mutationen in KRAS ebenfalls anerkannte Resistenzmechanismen auf eine EGFR-Blockade darstellen, und rund $40 \%$ der Kolontumore KRASMutationen aufweisen, ist KRAS ein negativer Prädiktor für eine Anti-EGFRTherapie. Daher wird bei Kolontumoren routinemäßig eine Bestimmung des KRAS-Mutationsstatus durchgeführt. Ähnlich wie bei Lungenkarzinomen ist die Ansprechrate von Patienten mit Kolorektalkarzinomen mit KRAS-WildtypTumoren anfangs sehr gut, allerdings kommt es auch hier in dem meisten Fällen nach sechs Monaten zur Progression. Wie bei den Lungenkrebspatienten können auch hier Resistenzmechanismen aufgrund von KRAS-Mutation oder Amplifikationen frühzeitig im Plasma detektiert werden $[98,99]$.

Mittlerweile gibt es auch Studien, welche die Verwendung von ctDNA zur Detektion von MRD bzw. Früherkennung von Rezidiven belegen, wofür sich aufgrund der hohen Sensitivität und Spezifität speziell der Nachweis von tumorspezifischen chromosomalen Umbauten eignet. Garcia-Murillas et al. konnten bei Patientinnen mit lokalisiertem Brustkrebs nach neoadjuvanter Therapie und Operation mit kurativer 
Intention mittels $\mathrm{dPCR}$ aus dem $\mathrm{Tu}$ mor etablierte Fusionsbruchpunkte im Schnitt acht Monate vor dem Auftreten eines Rezidivs im Plasma nachweisen. Im Gegensatz dazu konnten bei Patientinnen mit Mammakarzinom und einem Langzeit-progressionsfreien Überleben keine tumorspezifischen Veränderungen im Plasma detektiert werden [100]. Eine schwedische Gruppe lieferte ähnliche Ergebnisse: Das Vorhandensein von ctDNA, ebenfalls gemessen anhand spezifischer Fusionsbruchpunkte mit dPCR, erlaubte die Diskriminierung von Patientinnen mit Rezidiven und jenen mit Langzeit-progressionsfreiem Überleben, wobei das Auftreten der Rezidive bis zu zehn Monate vorhergesagt werden konnte [101].

\section{Klinischer Nutzen von um- fassenden Mutationsanalysen}

In vielen Tumorentitäten, beispielsweise bei Brustkrebs gibt es, im Gegensatz zu Kolon- und Lungentumoren oder auch dem Melanom, keine rekurrent auftretenden aktivierenden oder resistenzverleihenden Mutationen. Daher wird für ein effektives Therapiemonitoring solcher Entitäten eine umfassendere Analyse benötigt. Die Verwendung von Genpanels kann einerseits zu einer nicht invasiven Identifizierung von neu aufkommenden Therapiezielen beitragen, andererseits ermöglicht eine breite Genanalyse auch Einblicke in die $\mathrm{He}$ terogenität und Evolution der Tumore [102]. In einer Proof-of-Principle Studie haben De Mattos-Arrunda et al. 300 Gene bei einer Brustkrebspatientin (ER+/Her2) im Tumor, einer Lebermetastase sowie in der Plasma-DNA sequenziert [103]. Obwohl in der Metastase Mutationen gefunden wurden, die im Primärtumor nicht nachgewiesen werden konnten, reflektierte die ctDNA Aberrationen aus beiden Tumorgeweben [103]. Butler et al., konnten nach WES mehr Gemeinsamkeiten zwischen einer Lebermetastase und der ctDNA als mit dem Primärtumor finden, was für eine klonale Selektion unter der verabreichten Anastrozol Therapie spricht.
Während im Primärtumor eine PIK3CA Hotspot Mutation detektiert wurde, die im Plasma und der Metastase nicht nachgewiesen werden konnte, trat dort eine neue ESR1-Mutation auf, die mit großer Wahrscheinlichkeit für das Therapieversagen verantwortlich war [76]. Dieselbe Gruppe, die erstmalig WES aus Plasma publizierte, hat kürzlich einen Case Report veröffentlicht, indem sie bei einer metastasierten Brustkrebspatientin extensive Vergleiche der Mutationen in multiplen Biopsien und Plasmaproben anstellte. Die Ergebnisse zeigten, dass Plasma-DNA die Größe und Aktivität von unterschiedlichen Subklonen widerspiegelt und sogar ein differenzielles Therapieansprechen unterschiedlicher Metastasen reflektiert.

\section{Bedeutung von genomweiten Analysen}

Während die Entstehung und Progression einer Reihe von Tumoren eher von Mutationen in bestimmten onkogenen Pathways getrieben ist, findet man bei

\section{Hier steht eine Anzeige.}

\section{黑 Springer}


anderen Entitäten keine rekurrent mutierten Gene und auch insgesamt eine geringere Mutationslast. In diesen Tumoren findet sich häufig eine genomische Instabilität und daraus resultierend eine Reihe von SCNAs [104]. Daher ist hier eine Analyse von SCNAs für die Identifizierung von Resistenzmechanismen oder Therapiezielen von großer Bedeutung.

Bei Patienten mit Prostatakarzinom unter Hormontherapie konnte anhand von ctDNA-Analysen eine Assoziation zwischen dem Vorliegen von bestimmten SCNAs und dem klinischen Outcome gezeigt werden [105]. Die vermehrte Entstehung von fokalen Amplifikationen in fortgeschrittenen Stadien, was auf eine hohe Plastizität der Tumore hindeutet, konnte von uns kürzlich aus dem Plasma nachgewiesen werden [80]. Zudem konnten wir auch umfassende klonale Veränderungen in der Plasma-DNA beobachten, welche höchstwahrscheinlich auch mit einer Diversifizierung des Tumors einhergehen. Ähnliche Daten zeigen Carreiera et al., die im Tumorgewebe sowie in der Zirkulation mehrere unabhängige Klone detektieren konnten, die zu Therapieresistenzen beitragen [106].

Auch in anderen Entitäten unterliegen viele Resistenzmechanismen Kopienzahlveränderungen. Neben Mutationen in den Genen KRAS oder EGFR zeigen sich unter anderem auch fokale Amplifikationen von KRAS und MET bei progredienten Patienten unter Anti-EGFR-Therapie, die aus dem Plasma nachgewiesen werden können [86]. Diese und viele weitere Daten weisen auf eine äußerst komplexe Dynamik von Tumoren hin und unterstreichen die Notwendigkeit von kontinuierlich erfassten, genetischen Follow-up-Daten.

\section{Zusammenfassung und Ausblick}

Der technologische Fortschritt auf dem Gebiet der ctDNA Analytik hat eine breite Implementierung in die Klinik in greifbare Nähe gebracht. Das Potenzial von ctDNA als Biomarker ist unbestritten, da in unzähligen Studien der klinische Nutzen für ein verbessertes Therapiemanagement von Krebspatienten belegt wurde. Mittels genomweiter Analysen erlaubt die Analyse der ctDNA, insbesondere in fortgeschrittenen Stadien, ein umfassendes Therapiemonitoring, das es den behandelnden Ärzten erlaubt, möglichst rasch auf Veränderungen im Tumor zu reagieren und somit ihren Patienten teure Behandlungen mit teils sehr toxischen Medikamenten ab dem Augenblick zu ersparen, in dem diese ohnehin nicht mehr wirksam wären. Außerdem können neue Therapieziele, die im Laufe der Erkrankung auftreten, identifiziert werden und so dem Patienten zu neuen Therapiemöglichkeiten verhelfen. ctDNA bietet außerdem eine einzigartige Gelegenheit, mehr über Metastasierungsprozesse und die damit verbundenen Signalwege zu erfahren.

Anhand von hochauflösenden Methoden ist es möglich, ctDNA-Analysen auch schon in frühen Stadien, in denen der Anteil der Tumor-DNA in der Zirkulation oft nur sehr gering ist, durchzuführen und so frühzeitig das Auftreten eines Rezidivs vorherzusagen. Trotz all dieser methodischen Möglichkeiten fehlt es aber im Moment noch an etablierten internationalen Qualitätsstandards, wie und in welchem Umfang ctDNA-Analysen durchgeführt werden sollen. Die Notwendigkeit für einen Konsens über die präanalytischen und analytischen Strategien sowie die nachfolgende Befundung, Interpretation und Konsequenzen für Betroffene ist offensichtlich und entsprechende Konsortien wie beispielsweise das CancerID (http://www.cancer-id.eu/) befassen sich mit entsprechenden Anforderungen, um eine erfolgreiche Implementierung der Liquid Biopsy als validen klinischen Test voranzutreiben.

\section{Korrespondenzadresse}

Ass. Prof. PD Dr. rer. nat. E. Heitzer

Institut für Humangenetik, Medizinische Universität Graz

Harrachgasse 21/8, 8010 Graz, Österreich ellen.heitzer@medunigraz.at

Open access funding provided by Medical University of Graz.

\section{Einhaltung ethischer Richtlinien}

Interessenkonflikt. P. Ulz, J.B. Geigl, M.R. Speicher und $E$. Heitzer geben an, dass kein Interessenkonflikt besteht.

Dieser Beitrag beinhaltet keine von den Autoren durchgeführten Studien an Menschen oder Tieren.

Open Access Dieser Artikel wird unter der Creative Commons Namensnennung 4.0 International Lizenz (http://creativecommons.org/licenses/by/4.0/deed. de) veröffentlicht, welche die Nutzung, Vervielfältigung, Bearbeitung, Verbreitung und Wiedergabe in jeglichem Medium und Format erlaubt, sofern Sie den/die ursprünglichen Autor(en) und die Quelle ordnungsgemäß nennen, einen Link zur Creative Commons Lizenz beifügen und angeben, ob Änderungen vorgenommen wurden.

\section{Literatur}

1. Crowley E, Di Nicolantonio F, Loupakis F, Bardelli A (2013) Liquid biopsy: monitoring cancer-genetics in the blood. Nat Rev Clin Oncol 10(8):472-484

2. Diaz LA Jr., Bardelli A (2014) Liquid biopsies: genotyping circulating tumor DNA. J Clin Oncol 32(6):579-586

3. Heitzer E, Ulz P, Geigl JB (2015) Circulating tumor DNA as a liquid biopsy for cancer. Clin Chem 61(1):112-123

4. Schwarzenbach H, Hoon DS, Pantel K (2011) Cellfree nucleic acids as biomarkers in cancer patients. Nat Rev Cancer 11:426-437

5. Toth K, Bartak BK, Tulassay Z, Molnar B (2016) Circulating cell-free nucleic acids as biomarkers in colorectal cancer screening and diagnosis. Expert Rev MolDiagn 16(2):239-252

6. Bettegowda C, Sausen M, Leary RJ, Kinde I, Wang Y, Agrawal N et al (2014) Detection of circulating tumor DNA in early- and late-stage human malignancies. Sci TransI Med 6(224):224ra24

7. Amoura Z, Piette JC, Chabre H, Cacoub P, Papo T, Wechsler B et al (1997) Circulating plasma levels of nucleosomes in patients with systemic lupus erythematosus: Correlation with serum antinucleosome antibody titers and absence of clear association with disease activity. Arthritis Rheum 40(12):2217-2225

8. Rodrigues EM, Simon D, Ikuta N, Klovan C, Dannebrock FA, de Oliveira CO et al (2014) Elevated cell-free plasma DNA level as an independent predictor of mortality in patients with severe traumatic brain injury. J Neurotrauma 31(19):1639-1646

9. Zhong XY, von Muhlenen I, Li Y, Kang A, Gupta AK, Tyndall A et al (2007) Increased concentrations of antibody-bound circulatory cell-free DNA in rheumatoid arthritis. Clin Chem 53(9):1609-1614

10. Rainer TH, Wong LKS, Lam W, Yuen E, Lam NYL, Metreweli C et al (2003) Prognostic use of circulating plasma nucleic acid concentrations in patients with acute stroke. Clin Chem 49(4):562-569

11. Antonatos D, Patsilinakos S, Spanodimos $S$, Korkonikitas P, Tsigas D (2006) Cell-free DNA levels as a prognostic marker in acute myocardial infarction. Ann N Y Acad Sci 1075:278-281

12. Tamkovich SN, Cherepanova AV, Kolesnikova EV, Rykova EY, Pyshnyi DV, Vlassov VV et al (2006) Circulating DNA and DNase activity in human blood. Ann NY Acad Sci 1075:191-196 
13. Hawes MC, Wen F, Elquza E (2015) Extracellular DNA: A bridge to cancer. Cancer Res 75(20):4260-4264

14. Heitzer E, Auer M, Hoffmann EM, Pichler M, Gasch C,UlzPetal (2013) Establishment of tumor-specific copy number alterations from plasma DNA of patients with cancer. Int J Cancer 133(2):346-356

15. Lo YM, Chan KC, Sun H, Chen EZ, Jiang P, Lun FM et al (2010) Maternal plasma DNA sequencing reveals the genome-wide genetic and mutational profile of the fetus. Sci TransI Med 2(61):61 ra91

16. Diaz LA Jr., Williams RT, Wu J, Kinde I, Hecht JR, Berlin J et al (2012) The molecular evolution of acquired resistance to targeted EGFR blockade in colorectal cancers. Nature 486(7404):537-540

17. Snyder TM, Khush KK, Valantine HA, Quake SR (2011) Universal noninvasive detection of solid organ transplant rejection. Proc Natl Acad Sci USA 108(15):6229-6234

18. Lo YM, Tein MS, Pang CC, Yeung CK, Tong KL, Hjelm NM (1998) Presence of donor-specific DNA in plasma of kidney and liver-transplant recipients. Lancet 351(9112):1329-1330

19. Chan KC (2013) Cancer genome scanning in plasma: Detection of tumor-associated copy number aberrations, single-nucleotide variants, and tumoral heterogeneity by massively parallel sequencing. Clin Chem 59:211-224

20. Heitzer E, Auer M, Gasch C, Pichler M, Ulz P, HoffmannEMetal (2013)Complextumorgenomes inferred from single circulating tumor cells by array-CGH and next-generation sequencing. Cancer Res 73(10):2965-2975

21. Heitzer E, Ulz P, Belic J, Gutschi S, Quehenberger F, Fischereder K et al (2013) Tumor-associated copy number changes in the circulation of patients with prostate cancer identified through whole-genome sequencing. Genome Med 5(4):30

22. Spindler KL, Pallisgaard N, Vogelius I, Jakobsen A (2012) Quantitative cell-free DNA, KRAS, and BRAF mutations in plasma from patients with metastatic colorectal cancer during treatment with cetuximab and irinotecan. Clin Cancer Res 18:1177-1185

23. DiehI F, Li M, Dressman D, He Y, Shen D, Szabo Set al (2005) Detection and quantification of mutations in the plasma of patients with colorectal tumors. Proc Natl Acad SciUSA 102(45):16368-16373

24. Taly V, Pekin D, Benhaim L, Kotsopoulos SK, Le Corre D, Li Xet al (2013) Multiplex picodroplet digital PCR to detect KRAS mutations in circulating DNA from the plasma of colorectal cancer patients. Clin Chem 59(12):1722-1731

25. Diehl F, Schmidt K, Choti MA, Romans K, Goodman S, Li Metal (2008) Circulating mutant DNA to assess tumor dynamics. Nat Med 14(9):985-990

26. Douillard JY, Ostoros G, Cobo M, Ciuleanu T, Cole R, McWalter $\mathrm{G}$ et al (2014) Gefitinib treatment in EGFR mutated caucasian NSCLC: circulating-free tumor DNA as a surrogate for determination of EGFR status. JThorac Oncol 9(9):1345-1353

27. Aung KL, Donald E, Ellison G, Bujac S, Fletcher L, Cantarini $M$ et al (2014) Analytical validation of BRAF mutation testing from circulating free DNA using the amplification refractory mutation testing system. J Mol Diagn 16(3):343-349

28. Schreuer $M$, Meersseman $G$, van Den Herrewegen S, Jansen Y, Seremet T, Bott A et al (2016) Applications for quantitative measurement of BRAF V600 mutant cell-free tumor DNA in the plasma of patients with metastatic melanoma. Melanoma Res 26(2):157-163

29. Mouliere F, El Messaoudi S, Pang D, Dritschilo A, Thierry AR (2014) Multi-marker analysis of circula- ting cell-free DNA toward personalized medicine for colorectal cancer. Mol Oncol 8(5):927-941. doi:10.1016/j.molonc.2014.02.005

30. Mouliere F, El Messaoudi S, Gongora C, Guedj AS, Robert B, Del Rio M et al (2013) Circulating cell-free DNA from Colorectal cancer patients may reveal high KRAS or BRAF mutation load. Transl Oncol 6(3):319-328 (IN6-IN8) doi:10.1593/tlo.12445

31. Ashida A, Sakaizawa K, Mikoshiba A, Uhara H, Okuyama R (2016) Quantitative analysis of the BRAF mutation in circulating tumor-derived DNA in melanoma patients using competitive allelespecific TaqMan PCR. Int J Clin Oncol. doi:10.1007/ s10147-016-0976-y

32. Bolton L, Reiman A, Lucas K, Timms J, Cree IA (2015) KRAS mutation analysis by PCR: a comparison of two methods. PLoSONE 10(1):e0115672

33. Stadler J, Eder J, Pratscher B, Brandt S, Schneller $D$, Mullegger R et al (2015) SNPase-ARMS qPCR: ultrasensitive mutation-based detection of cellfree tumor DNA in melanoma patients. PLoS ONE 10(11):e0142273

34. Madic J, Piperno-Neumann S, Servois V, Rampanou A, Milder M, Trouiller B et al (2012) Pyrophosphorolysis-activated polymerization detects circulating tumor DNA in metastatic uveal melanoma. Clin Cancer Res 18(14):3934-3941

35. Bidard FC, Madic J, Mariani P, Piperno-Neumann S, Rampanou A, Servois V et al (2014) Detection rate and prognostic value of circulating tumor cells and circulating tumor DNA in metastatic uveal melanoma. Int J Cancer 134(5):1207-1213

36. Dabritz J, Hanfler J, Preston R, Stieler J, Oettle $H$ (2005) Detection of Ki-ras mutations in tissue and plasma samples of patients with pancreatic cancer using PNA-mediated PCR clamping and hybridisation probes. Br J Cancer 92(2):405-412

37. Freidin MB, Freydina DV, Leung $M$, Montero Fernandez A, Nicholson AG, Lim E (2015) Circulating tumor DNA outperforms circulating tumor cells for KRAS mutation detection in thoracic malignancies. Clin Chem 61(10):1299-1304

38. Milbury CA, Chen CC, Mamon H, Liu P, Santagata $S$, Makrigiorgos GM (2011) Multiplex amplification coupled with COLD-PCR and high resolution melting enables identification of low-abundance mutations in cancer samples with low DNA content. J Mol Diagn 13(2):220-232

39. Pinzani P, Santucci C, Mancini I, Simi L, Salvianti F, Pratesi $\mathrm{N}$ et al (2011) BRAF(V600E) detection in melanoma is highly improved by COLD-PCR. Clin Chim Acta 412(11-12):901-905

40. Guha M, Castellanos-Rizaldos E, Liu PF, Mamon H, Makrigiorgos GM (2013) Differential strand separation at critical temperature: a minimally disruptive enrichment method for low-abundance unknown DNA mutations. Nucleic Acids Res 41:3

41. Guha M, Makrigiorgos M (2012) Differential strand separation at critical temperature reveals lowabundance mutations in cancer samples. J Mol Diagn 14(6):699-700

42. Hu C, Liu X, Chen Y, Sun X, Gong Y, Geng M et al (2012) Direct serum and tissue assay for EGFR mutation in non-small cell lung cancer by high-resolution melting analysis. Oncol Rep 28(5):1815-1821

43. Jing CW, Wang Z, Cao HX, Ma R, Wu JZ (2014) High resolution melting analysis for epidermal growth factor receptor mutations in formalin-fixed paraffin-embedded tissue and plasma free DNA from non-small cell lung cancer patients. Asian Pac J Cancer Prev 14(11):6619-6623

44. Pinzani P, Salvianti F, Cascella R, Massi D, De Giorgi V, Pazzagli M et al (2010) Allele specific Taqman- based real-time PCR assay to quantify circulating BRAFV600E mutated DNA in plasma of melanoma patients. Clin Chim Acta 411(17-18):1319-1324

45. Chen D, Huang JF, Xia H, Duan GJ, Chuai ZR, Yang $Z$ et al (2014) High-sensitivity PCR method for detecting BRAF V600E mutations in metastatic colorectal cancer using LNA/DNA chimeras to block wild-type alleles. Anal Bioanal Chem 406(9-10):2477-2487

46. Bai H, Mao L, Wang HS, Zhao J, Yang L, An TT et al (2009) Epidermal growth factor receptor mutations in plasma DNA samples predict tumor response in Chinese patients with stages IIIB to IV non-small-cell lung cancer. J Clin Oncol 27(16):2653-2659

47. Brevet M, Johnson ML, Azzoli CG, Ladanyi M (2011) Detection of EGFR mutations in plasma DNA from lung cancer patients by mass spectrometry genotyping is predictive of tumor EGFR status and response to EGFR inhibitors. Lung Cancer 73:96-102

48. Mosko MJ, Nakorchevsky AA, Flores E, Metzler $H$, Ehrich M, van den Boom DJ et al (2016) Ultrasensitive detection of multiplexed somatic mutations using MALDI-TOF mass spectrometry. JMol Diagn 18(1):23-31

49. Baker M (2012) Digital PCR hits its stride. Nat Methods 9(6):541-544

50. Day E, Dear PH, McCaughan F (2013) Digital PCR strategies in the development and analysis of molecular biomarkers for personalized medicine. Methods 59(1):101-107

51. Higgins MJ, Jelovac D, Barnathan E, Blair B, Slater S, Powers P et al (2012) Detection of tumor PIK3CA status in metastatic breast cancer using periphera blood. Clin Cancer Res 18(12):3462-3469

52. Chang GA, Tadepalli JS, Shao Y, Zhang Y, Weiss $S$, Robinson E et al (2016) Sensitivity of plasma BRAFmutant and NRASmutant cell-free DNA assays to detect metastatic melanoma in patients with low RECIST scores and non-RECIST disease progression. MolOncol 10(1):157-165

53. Shoda K, Ichikawa D, Fujita Y, Masuda K, Hiramoto $\mathrm{H}$, Hamada J et al (2016) Monitoring the HER2 copy number status in circulating tumor DNA by droplet digital PCR in patients with gastric cancer. Gastric Cancer. doi:10.1007/s10120-016-0599-z

54. Leary RJ, Kinde I, Diehl F, Schmidt K, Clouser C, Duncan C et al (2010) Development of personalized tumor biomarkers using massively parallel sequencing. Sci Transl Med 2:20

55. Leary RJ, Sausen M, Kinde I, Papadopoulos N, Carpten JD, Craig D et al (2012) Detection of chromosomal alterations in the circulation of cancer patients with whole-genome sequencing. Sci Transl Med 4(162):162ra54

56. Narayan A, Carriero NJ, Gettinger SN, Kluytenaar J, Kozak KR, Yock TI et al (2012) Ultrasensitive measurement of hotspot mutations in tumor DNA in blood using error-suppressed multiplexed deep sequencing. Cancer Res 72(14):3492-3498

57. Kukita Y, Uchida J, Oba S, Nishino K, Kumagai T, Taniguchi Ket al (2013) Quantitative identification of mutant alleles derived from lung cancer in plasma cell-free DNA via anomaly detection using deep sequencing data. PLoSONE 8(11):e81468

58. Uchida J, Kato K, Kukita Y, Kumagai T, Nishino $\mathrm{K}$, Daga $\mathrm{H}$ et al (2015) Diagnostic accuracy of noninvasive genotyping of EGFR in lung cancer patients by deep sequencing of plasma cell-free DNA.Clin Chem 61(9):1191-1196

59. Jabara CB, Jones CD, Roach J, Anderson JA, Swanstrom R (2011) Accurate sampling and deep sequencing of the HIV-1 protease gene 
using a Primer ID. Proc Natl Acad Sci USA 108(50):20166-20171

60. Kinde I, Wu J, Papadopoulos N, Kinzler KW, Vogelstein B (2011) Detection and quantification of rare mutations with massively parallel sequencing. Proc Natl Acad Sci USA 108(23):9530-9535

61. Schmitt MW, Kennedy SR, Salk JJ, Fox EJ, Hiatt JB, Loeb LA (2012) Detection of ultra-rare mutations by next-generation sequencing. Proc Natl Acad Sci USA 109(36):14508-14513

62. Newman AM, Lovejoy AF, Klass DM, Kurtz DM, Chabon JJ, Scherer Fet al (2016) Integrated digital error suppression for improved detection of circulating tumor DNA. Nat Biotechnol 34(5):547-555

63. Lanman RB, Mortimer SA, Zill OA, Sebisanovic D, Lopez R, Blau S et al (2015) Analytical and clinical validation of a digital sequencing panel for quantitative, highly accurate evaluation of cell-free circulating tumor DNA. PLoSONE 10(10):e0140712

64. National Comprehensive Cancer Network (NCCN) (2014) Guidelines for non-small cell lung cancer (NSCLC) (4.2014 N-SCLCV)

65. Martinez P, McGranahan N, Birkbak NJ, Gerlinger $M$, Swanton C (2013) Computational optimisation of targeted DNA sequencing for cancer detection. Sci Rep 3:3309

66. Ong M, Carreira S, Goodall J, Mateo J, Figueiredo I, Rodrigues DN et al (2014) Validation and utilisation of high-coverage next-generation sequencing to deliver the pharmacological audit trail. Br J Cancer 111(5):828-836

67. Xu S, Lou F, Wu Y, Sun DQ, Zhang JB, Chen W et al (2016) Circulating tumor DNA identified by targeted sequencing in advanced-stage nonsmall cell lung cancer patients. Cancer Lett 370(2):324-331

68. Jovelet C, lleana E, Le Deley MC, Motte N, Rosellini $S$, Romero A et al (2016) Circulating cell-free tumor DNA analysis of 50 genes by next-generation sequencing in the prospective MOSCATO trial. Clin Cancer Res. doi:10.1158/1078-0432.ccr-15-2470

69. Forshew T, Murtaza M, Parkinson C, Gale D, Tsui DW, Kaper F et al (2012) Noninvasive identification and monitoring of cancer mutations by targeted deep sequencing of plasma DNA. Sci Transl Med 4(136):136ra68

70. Dawson SJ (2013) Analysis of circulating tumor DNA to monitor metastatic breast cancer. $N$ Engl J Med 368:1199-1209

71. Drilon A, Wang L, Arcila ME, Balasubramanian S, Greenbowe JR, Ross JS et al (2015) Broad, hybrid capture-based next-generation sequencing identifies actionable genomic alterations in lung adenocarcinomas otherwise negative for such alterations by other genomic testing approaches. Clin Cancer Res 21(16):3631-3639

72. Paweletz CP, Sacher AG, Raymond CK, Alden RS, O'Connell A, Mach SL et al (2016) Bias-corrected targeted next-generation sequencing for rapid, multiplexed detection of actionable alterations in cell-free DNA from advanced lung cancer patients. Clin Cancer Res 22(4):915-922

73. Newman AM, Bratman SV, To J, Wynne JF, Eclov NCW, Modlin LA et al (2014) An ultrasensitive method for quantitating circulating tumor DNA with broad patient coverage. Nat Med 20(5):548-554. doi:10.1038/nm.3519

74. Murtaza M, Dawson SJ, Tsui DW, Gale D, Forshew T, Piskorz AM et al (2013) Non-invasive analysis of ac quired resistance to cancer therapy by sequencing of plasma DNA. Nature 497(7447):108-112

75. Heidary M, Auer M, Ulz P, Heitzer E, Petru E, Gasch C et al (2014) The dynamic range of circulating tumor DNA in metastatic breast cancer. Breast Cancer Res 16(4):421
76. Butler TM, Johnson-Camacho K, Peto M, Wang NJ, Macey TA, Korkola JE et al (2015) Exome sequencing of cell-free DNA from metastatic cancer patients identifies clinically actionable mutations distinct from primary disease. PLOSONE 10(8):e0136407

77. Jamal-Hanjani M, Wilson GA, Horswell S, Mitter $\mathrm{R}_{t}$ Sakarya O, Constantin T et al (2016) Detection of ubiquitous and heterogeneous mutations in cellfree DNA from patients with early-stage non-smallcell lung cancer. Ann Oncol 27(5):862-867

78. Pereira E, Camacho-Vanegas O, Anand S, Sebra R, Catalina Camacho S, Garnar-Wortzel L et al (2015) Personalized circulating tumor DNA biomarkers dynamically predict treatment response and survival in gynecologic cancers. PLoS ONE 10(12):e0145754

79. Chan KC, Jiang P, Chan CW, Sun K, Wong J, Hui EPetal (2013) Noninvasive detection of cancer-associated genome-wide hypomethylation and copy number aberrations by plasma DNA bisulfite sequencing. Proc Natl Acad Sci USA 110(47):18761-18768

80. Ulz PBJ, Graf R, Auer M, Lafer I, Fischereder K, Webersinke G, Pummer K, Augustin $\mathrm{H}$, Pichler M, Hoefler G, Bauernhofer T, Geigl JB, Heitzer E, Speicher MR (2016) Whole-genome plasma sequencing reveals focal amplifications as driving force in metastatic prostate cancer. Nat Commun 7:12008. doi:10.1038/ncomms12008

81. Beroukhim R, Mermel CH, Porter D, Wei G, Raychaudhuri S, Donovan J et al (2010) The landscape of somatic copy-number alteration across human cancers. Nature 463(7283):899-905

82. Carter SL, Eklund AC, Kohane IS, Harris LN, Szallasi Z (2006) A signature of chromosomal instability inferred from gene expression profiles predicts clinical outcome in multiple human cancers. Nat Genet 38(9):1043-1048

83. Zack TI, Schumacher SE, Carter SL, Cherniack AD, Saksena G, TabakB et al (2013) Pan-cancer patterns of somatic copy number alteration. Nat Genet 45(10):1134-1140

84. Heitzer E, Ulz P, Geigl JB, Speicher MR (2016) Noninvasive detection of genome-wide somatic copy number alterations by liquid biopsies. Mol Oncol 10(3):494-502

85. Shaw JA, Page K, Blighe K, Hava N, Guttery D, Ward B et al (2012) Genomic analysis of circulating cellfree DNA infers breast cancer dormancy. Genome Res 22(2):220-231

86. Mohan S, Heitzer E, Ulz P, Lafer I, Lax S, Auer Met al (2014) Changes in colorectal carcinoma genomes under anti-EGFR therapy identified by wholegenome plasma DNA sequencing. PLoS Genet 10(3):e1004271

87. Ulz PAM, Graf R, Kashofer K, Jahn SW, Abete L, Pristauz G, Petru E, Geigl JB, Heitzer E, Thallinger GG, Speicher MR (2016) Inferring expressed genes by whole-genome sequencing of plasma DNA. Nat Genet. doi:10.1101/049478

88. Belic J, Koch M, Ulz P, Auer M, Gerhalter T, Mohan S et al (2015) Rapid identification of plasma DNA samples with increased ctDNA levels by a modified FAST-seqSapproach. Clin Chem 61(6):838-849

89. Thunnissen E, van der Oord K, den Bakker M (2014) Prognostic and predictive biomarkers in lung cancer. A review. Virchows Arch 464(3):347-358

90. Rosell R, Moran T, Queralt C, Porta R, Cardenal F, Camps C et al (2009) Screening for epidermal growth factor receptor mutations in lung cancer. $\mathrm{N}$ Engl JMed 361(10):958-967

91. Chan BA, Hughes BG (2015) Targeted therapy for non-small cell lung cancer: current standards and the promise of the future. Transl Lung Cancer Res 4(1):36-54

92. Tsui DW, Berger MF (2016) Profiling non-small cell lung cancer: from tumor to blood. Clin Cancer Res 22(4):790-792

93. Tissot C, Villar S, Olivier M, Couraud S (2016) Free circulating DNA as a tool for lung cancer patients management. RevPneumolClin 72(1):61-71

94. Taniguchi K, Uchida J, Nishino K, Kumagai T, Okuyama T, Okami J et al (2011) Quantitative detection of EGFR mutations in circulating tumor DNA derived from lung adenocarcinomas. Clin CancerRes 17(24):7808-7815

95. Sacher AG, Paweletz C, Dahlberg SE, Alden RS O'Connell A, Feeney $\mathrm{N}$ et al (2016) Prospective validation of rapid plasma genotyping for the detection of EGFR and KRAS mutations in advanced lung cancer. JAMA Oncol. doi:10.1001/ jamaoncol.2016.0173

96. Sundaresan TK, Sequist LV, Heymach JV, Riely GJ, JannePA, Koch WHetal (2016) Detection of T790M, the acquired resistance EGFR mutation, by tumor biopsy versus noninvasive blood-based analyses. Clin Cancer Res 22(5):1103-1110

97. Yanagita M, Redig AJ, Paweletz CP, Dahlberg SE, O'Connell A, Feeney N et al (2016) A prospective evaluation of circulating tumor cells and cell-free DNA in EGFR mutant non-small cell lung cancer patients treated with erlotinib on a phase II trial. Clin Cancer Res. doi:10.1158/1078-0432.ccr-160909

98. Bronte G, Silvestris N, Castiglia M, Galvano A Passiglia F, Sortino G et al (2015) New findings on primary and acquired resistance to anti-EGFR therapy in metastatic colorectal cancer: Do all roads lead to RAS? Oncotarget 6(28):24780-24796

99. Morelli MP, Overman MJ, Dasari A, Kazmi SM Mazard T, Vilar E et al (2015) Characterizing the patterns of clonal selection in circulating tumor DNA from patients with colorectal cancer refractory to anti-EGFR treatment. Ann Oncol 26(4):731-736

100. Garcia-Murillas I, Schiavon G, Weigelt B, Ng C Hrebien S, Cutts RJ et al (2015) Mutation tracking in circulating tumor DNA predicts relapse in early breast cancer. Sci TransI Med 7:302

101. Olsson E, Winter C, George A, Chen Y, Howlin J, Tang $\mathrm{MH}$ et al (2015) Serial monitoring of circulating tumor DNA in patients with primary breast cancer for detection of occult metastatic disease. EMBO Mol Med 7(8):1034-1047. doi:10.15252/emmm. 201404913

102. Aparicio S, Caldas C (2013) The implications of clonal genome evolution for cancer medicine. $\mathrm{N}$ Engl J Med 368(9):842-851

103. De Mattos-Arruda L, Weigelt B, Cortes J, Won HH, $\mathrm{Ng}$ CKY, Nuciforo P et al (2014) Capturing intratumor genetic heterogeneity by de novo mutation profiling of circulating cell-free tumor DNA: a proof-of-principle. Ann Oncol 25(9):1729-1735

104. Ciriello G, Miller ML, Aksoy BA, Senbabaoglu $Y$, Schultz N, Sander C (2013) Emerging landscape of oncogenic signatures across human cancers. Nat Genet 45(10):1127-1133

105. Salvi S, Casadio V, Conteduca V, Burgio SL, Menna C, Bianchi E et al (2015) Circulating cell-free AR and CYP17A1 copy number variations may associate with outcome of metastatic castration-resistant prostate cancer patients treated with abiraterone. Br JCancer 112(10):1717-1724

106. Carreira S, Romanel A, Goodall J, Grist E, Ferraldeschi R, Miranda S et al (2014) Tumor clone dynamics in lethal prostate cancer. Sci Transl Med 6(254):254ra125 